\title{
Developing a Conceptual Design Engineering Toolbox and its Tools
}

\author{
R. W. Vroom, E. J. J. van Breemen, W. F. van der Vegte
}

In order to develop a successful product, a design engineer needs to pay attention to all relevant aspects of that product. Many tools are available, software, books, websites, and commercial services. To unlock these potentially useful sources of knowledge, we are developing C-DET, a toolbox for conceptual design engineering. The idea of C-DET is that designers are supported by a system that provides them with a knowledge portal on one hand, and a system to store their current work on the other. The knowledge portal is to help the designer to find the most appropriate sites, experts, tools etc. at a short notice. Such a toolbox offers opportunities to incorporate extra functionalities to support the design engineering work. One of these functionalities could be to help the designer to reach a balanced comprehension in his work. Furthermore C-DET enables researchers in the area of design engineering and design engineers themselves to find each other or their work earlier and more easily. Newly developed design tools that can be used by design engineers but have not yet been developed up to a commercial level could be linked to by C-DET. In this way these tools can be evaluated in an early stage by design engineers who would like to use them. This paper describes the first prototypes of C-DET, an example of the development of a design tool that enables designers to forecast the use process and an example of the future functionalities of C-DET such as balanced comprehension.

Keywords: toolbox, conceptual design engineering, development process, use process forecast, balanced comprehension.

\section{Introduction}

Design engineering is a knowledge and information intensive activity. However, there are many design tools, the Internet and digital libraries containing publications on almost every subject. We suffer from the enormous amount of available information, tools and knowledge that make the search for the most useful bits a time-consuming activity. We are exploring this problem area and have found that design students have problems finding appropriate components for their designs. For example one student searched on the Internet for about eight hours without finding a proper motor for his design of a hairdryer. Furthermore, design engineers in practice use the Internet for gaining knowledge, but many of them do not have a systematic approach that helps them to find the knowledge quickly and effectively. Another problem is that although many tools have been developed or are under development to help the designer to optimize the speed, quality or cost of his work, design engineers are often not aware of the existence of these tools, nor do they have the time to regularly scan the market in order to discover them. Finally, design engineers not only look for information and knowledge, they also create it when designing new products. We have not encountered existing tools that help the designer in the early phases of design to get an overview of his work in order to see what items and aspects have been covered yet and which are still missing or insufficiently handled. In order to overcome information and knowledge search and process problems of industrial design engineers, we aim to develop of a conceptual design engineering toolbox (C-DET) in which existing tools, links, information and knowledge are made available for designers at an appropriate point in time and place in their conceptual development process and which helps designers to document and structure the output of their conceptual design work.

This paper, begins by presenting the development of the early prototypes of C-DET. Then, the development of a tool that fits very well into C-DET is described as an example of tool development that is monitored closely for the up-to-date framework-function of C-DET. This is a tool that supports the designer in forecasting the usage of his product during conceptual design. Finally, 'extra functionality' that can be incorporated in C-DET is discussed, for example helping the designer to reach a balanced comprehension in his work. This extra functionality could be incorporated after the framework-function of C-DET is running properly.

\section{The development of C-DET}

In the early 1990s, several research programs aimed at developing some kind of integrated design environment. The ambition for these programs was very high [1]. Mostly the aim was to build an integrated database in which all output could be stored in a way that all people concerned could get their (part of the) information in a format that they needed. To achieve this, all kinds of mappings between different kinds of information were required. Some of these mappings are still unavailable [2]. That is why the goal for C-DET is to function as a framework for all kinds of useful bits rather than functioning as a comprehensive tool in which everything is linked through an integrated database.

The starting point for developing C-DET is that, to some extent design engineers work systematically (for example they orientate on the design problem, they make a list of requirements, they follow some design process) and that design engineers use their own intelligence. In other words, they are able to design and develop products for people. C-DET will only support them in their work. For example, by making knowledge and tools and knowledge about tools better available and by helping them with structuring the work and by giving overview of the work done. These functionalities can have some level of intelligence but will never ignore the intelligence of the design engineer.

\subsection{Objectives}

The goal of this research is to develop a toolbox for conceptual design engineering (C-DET) in which existing tools, 
links, information and knowledge are made available for designers at an appropriate point (in time and place) in their conceptual development process, and which helps the designer to document and structure the output of his conceptual design phase. Howevwr, the aim is not to create a central integrated database that is able to convert and translate all kind of data from one tool to another. Tools to be integrated vary from planning tools to CAD-tools, simulation tools, etc. Interaction between the researchers, developers and users of C-DET should lead to a useful toolbox in which design engineers properly rate links, tools, knowledge, etcetera, for use during the concept phase. Monitoring the use of C-DET and active rating enhances the usefulness.

The idea for C-DET is that the designer chooses which phase or what aspect he wants to work at. After that selection the designer can select helpful tools and links specifically for that phase or aspect. He can also create and/or insert his own specific design results for that phase or aspect. The way the output of his design project is structured and documented will be in an early prototype similar to the way that our design students make their design reports.

\subsection{Approach}

The approach is both theoretical and practical. C-DET will be based on a theoretical framework including for example: the descriptions of conceptual design described in [2], the methods, techniques and methodologies used in design education at the Faculty of Industrial Design Engineering at Delft, e.g. described in [3], the conceptual development part of the induced model in [4], other papers, e.g. [5], in which some categorizations of knowledge are presented.

The practical track is intuitive, trial and error, but supported with prototypes and experiments. The early prototypes of C-DET (will) have a small kernel, only serving a small design domain. This can be extended to the limits to maintain a balance between creating a toolbox that is too comprehensive (another internet/world) and a toolbox that is too small (not useful).

The early prototypes of C-DET will be mainly focused on the knowledge portal-function. Research will cover items such as the way the knowledge, tools, etc. should be structured and made accessible (interface and entrance structure) in order to optimally support design engineers in finding the information they need. We foresee that a single way of categorizing knowledge will not be sufficient. For example, in order to support the design engineer in structuring his output we need a structure according to design process phases and, in order to enable some support in balanced comprehension (see later section) of a design result, we need a structure including design aspects (such as ergonomics, manufacturability, etc.). Furthermore, in a parallel project carried out by Restrepo ([6, 7]), a prototype is created in which information is structured according to product domain, such as bikes and offices, which might be another entrance structure in C-DET. Furthermore we expect that finding knowledge, tools etc. by using keywords would also be very valuable for designers. In two prototypes of the C-DET system we have established two approaches in making available the tools and knowledge of our system. One approach is to link tools and knowledge sources to a phase structure, so designers can search and browse for knowledge using the phase they are in as guidance to the sought knowledge. The other approach is based on the designer's notion of the specific aspect that he/she is working on. We are investigating the appropriateness of these approaches. Both prototypes are discussed briefly in this paper.

Besides the entrance structure we need to find links, knowledge, tools etc. to be made available within C-DET. For this we need criteria, a rating system and many search hours (by students). C-DET will function as a framework in which existing material (including tools newly developed by colleagues or others) is made available. This way C-DET might be able to visualize the gaps in existing design support.

In the early stages of developing C-DET we also need to investigate in what way the communication with design engineers - more specifically their feedback on the links, tools and knowledge - should be incorporated. This feedback will be used to improve C-DET, to improve the criteria used for selecting knowledge, tools etc., to improve the ratings and/or the rating system and also to evaluate the newly developed tools that are integrated in C-DET.

Another topic that needs attention in the early stages of C-DET is the way the work of users could be inserted and stored. The output structure of this work needs to be developed and evaluated. The idea is that when the design work is inserted in C-DET, it can be printed in such a way that the designer has an intermediate or final design report presenting his conceptual design stage. For this we need to differentiate between public and private data.

C-DET might be able to support cooperation between design engineers, even when these engineers are not at the same location. The required functionality for this kind of support needs further investigation.

\subsection{Progress: two prototypes}

The first two prototypes we have made deal only with the knowledge portal part of C-DET. The goal for the prototype was to create a knowledge portal for some 100 websites and tools that can be used during the conceptual design process in order to evaluate our initial ideas about C-DET [8]. The entrance structure should be some kind of basic design process. The questions to be answered in this part are: Which phases are walked through by design engineers during the design process and what are the most common names for each of these phases; Which sites and tools are relevant for design engineers; How should these sites and tools be categorized and presented.

The first question is taken care of by studying literature e.g. [3, 4]. Design engineers have their own way of working, and the methods and techniques used vary enormously between design engineers and between design projects. Since it seems impossible to set up one concrete design process for all methods, we decided to set up an extensive design process in which most phases can be found. Not in every design project will all phases be walked through. Of course design engineers using C-DET will not be forced to do so. The design engineer himself determines in which phase of his design process he is. The common basis of the design processes described in the literature is used, and is supplemented by relevant steps described in design methods and techniques. In this way the following basic design process has been put forward: 
- Phase 1: Problem analysis (Information phase, Orientation phase, Goal finding). Sub phases are: lay down outline timetables; clarify and define the task; acquire general information; analyse the user environment; analyse usage; analyse competitive products; form a vision (collages); create a life cycle analysis; determine the list of requirements.

- Phase 2: Conceptual design (Product design phase). Sub-phases are: start with the first sketches; evaluate and choose from ideas; possible clustering of ideas; search for solution principles; combine into concept variants; determine specifications of the concept variants; compare concept variants and choose from them; finally make a solution concept.

- Phase 3: Embodiment design (Detail design, Optimisation phase). Sub-phases are: Summarise aspects still to be improved; improve specific aspects where needed; conduct studies into possible forms and shapes; conduct studies into possible colours; apply ergonomics data; investigate materials; investigate electronics; investigate environmental consequences; conduct mechanical calculations; develop plans for production and assembly; create an exploded-view; generate costs optimisation; optimise and complete the form design; put together a definitive design; construct a plan for building the prototype).

- Phase 4: Presentation. Sub-phases are: generate design documentation; create rendering; build a prototype.
- Phase 5: Evaluation. Sub-phases are: test the prototype (user trials); test by list of requirements; evaluate the process and its results; make recommendations for adjustments; prepare for production.

The initial contents (sites and tools) for the toolbox are collected in three ways: using a questionnaire for our design students; searching literature that is referenced in our courses; searching the Internet. The initial categorization is based on our own experience and knowledge and will be evaluated by tested using the prototype. The first prototype is created in ASP. In the use test we that we carried out with this prototype, only design students participated. We asked the students to carry out a small design task in which some search items are included. The results of this use test are promising. Users were enthusiastic about the knowledge portal. They were able to find information and tools and they began "playing" with the prototype because of the interesting links they found. However the entrance structure exclusively based on design phases was not considered optimal.

To gain more indications about an entrance structure based on design aspects, we set up a questionnaire. Based on internal reports e.g. [9] and the literature [3], [10] the following design aspects are expected to be important for design engineering projects: Technology/Technical aspects; Materials; Manufacturing; Design/Aesthetics; Ergonomics; Management Science; Ecology; Regulations; Marketing.
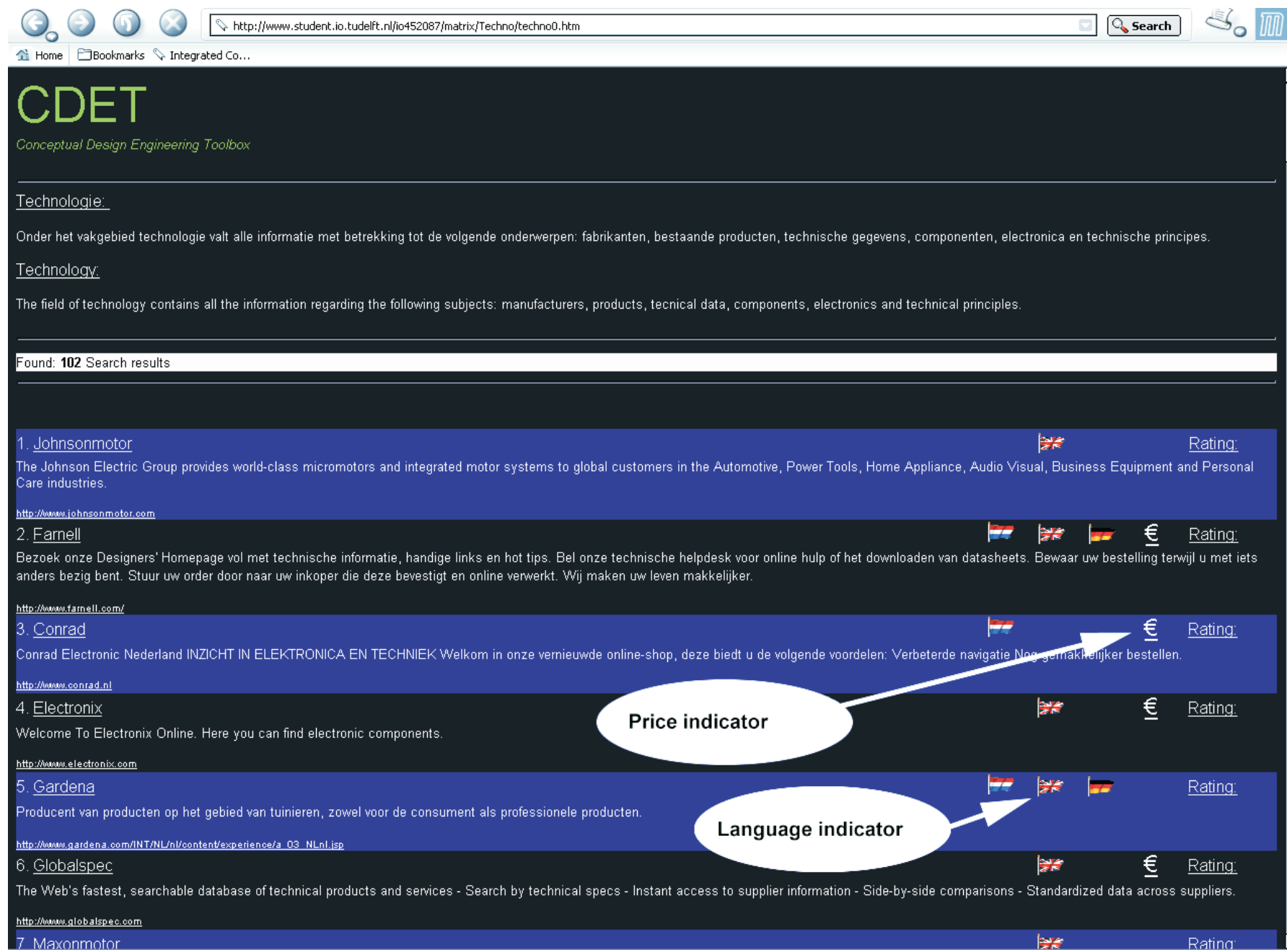

Fig. 1: First prototype of C-DET 
Furthermore we wanted to evaluate the criteria used for selecting websites and tools. These criteria are based on [8], $[10,11]$ and are: Advertisements and links are not the only information on the site; It is a non-commercial site; There is an option for requesting more specific information; Links to enable further searching are present; The site includes a search engine; Easy way of navigating within the site; The site provides a guest book (or forum); The site suggests that a serious effort was put into the aesthetics of the site; Technical pictures support the text; The site contains moving animations (e.g. Flash intros); The time required for loading the site is short; A notification of the last update of the site is present; The last update was performed recently; The available links are all still present and "alive". Based on the answers to the questionnaire, two criteria are skipped, namely the criterion about the guest book and about the moving animations. Furthermore, for our user group, the language in which the site is presented is limited to Dutch and English.

In the second prototype [12] the entrance structure is a matrix consisting of design phases (rows) and design aspects (columns). Some 400 sites are linked. On the page of the search results we have added two features, based on answers of respondents and user tests of the first prototype: a price indicator and a language indicator. See Fig. 1.

For the use test with the second prototype of C-DET we set up twelve search assignments that a testes has to carry out within 10 minutes, after a short introduction to the toolbox. The search assignments are for example: You are making a cover for a presentation or a design report and you want to gain some ideas and inspiration; You want to know the recycling options and the environmental impact of a material that you are using in a product design (e.g. polyethylene); You are looking for a component in Solid Works format to insert in your own CAD-drawing; 4) You want to know what material is most appropriate to use in your design of a MP3 player; etcetera.

Fifteen users participated in the user tests. The users did not understand the principle of C-DET right away. However, once they had used the toolbox for only a few minutes, their insight into the entrance structure increased enormously, which resulted in faster results of the search assignments. Some users would have categorized the sites slightly differently than we did. Not all search assignments were carried out successfully. That is because some subjects could be found at multiple aspects. This causes doubt about the button where to use for searching for the information.

\section{Development of the use-forecast tool}

Probably the most crucial phase in a product life cycle is the stage in which the product is used by humans to fulfil its intended functions. One of the issues is how to include knowledge related to the use stage of products in computer-aided conceptual design, as a supplement to functional modelling, artefact modelling and artefact-behaviour modelling. This issue has to be considered in the context of the increasing deployment of knowledge-intensive systems in computer support of design. The two key elements of our approach are (1) deployment of ontologies as a knowledge repository and (2) introducing knowledge-intensiveness into models by applying a nucleus-based approach. The application of ontologies has proven to be an advantageous paradigm in the research field of knowledge representation and knowledge processing . The nucleus-based modelling approach makes it possible to apply the ontology paradigm to so-called design concepts that offer integrated support to the product designer for modelling artefact geometry and artefact behaviour, in close connection to the artefact's function and its intended use by humans.

\subsection{Ontology-based support of usage consideration in conceptual design}

For the application of ontologies in our project, cooperation was initiated with experts in the field of functional-ontology modelling at the Department of Knowledge Systems at Osaka University, Japan. The objectives of functional-ontology modelling are

a) to provide insight into the design rationale by making the intended product behaviour explicit and

b) to provide computer-generated suggestions for alternatives based on the given functions in a product.

The collaborative project aims to extend towards use-process modelling, with a specific focus on forecasting the different use processes that are possible if the behaviour of user $U$ and environment $E$ are also to be taken into account. Unlike the design of product $P$, the $U$ and $E$ are not likely to be changed by the designer. A product on its own may sometimes not behave as was intended by the designer, but the risk that $U$ or $E$ does not behave as was intended, causing unwanted effects, is even more plausible. Moreover, unintended behaviour of $P$ itself is likely to be prompted by $U$ or $E$. Therefore, we want to offer assistance in managing the knowledge about possible (i.e., intended and unintended) use processes so that designers can anticipate them. This knowledge can originate from various sources, such as simulations, insight gained from previous products, or data collected from interactive user participation sessions. Needless to say, it will not be realistic to capture and manage knowledge about all possible use processes, but we do not strive to exclude any particular use apriori. So far, the research into deployment of ontologies for the consideration of use processes has been at an explorative level, and it has not reached the stage that its results can be applied or tested by designers in the form of computer support. Nevertheless, the exploration of use-related knowledge resulted in categorizations of

a) typical and atypical forms of unintended use,

b) forms of possible computer support, ranging from finding unintended use to solving the problems it causes,

c) principal approaches that can be taken to solve the problems.

C-DET offers an attractive opportunity to present these results to designers so that they can use them for guidance in their own approach to use consideration, and for gathering feedback about the prospect of proposed forms of computer support.

\subsection{Knowledge-intensive models}

In the early stage of design, designers typically create the first concrete models of products. Unfortunately, the modelling techniques and representations currently used in analysis and behavioural simulation do not provide effective means 
for modelling the use of products. One of the reasons is that in modelling use, not only $P$ has to be modelled, but also $U$ and $E$. The concrete problems with the available geometry-oriented modelling environments and simulation tools are:

1) the created models are supposed to be complete and valid, which is not necessary in conceptual design;

2) the simulations are orientated toward the behaviour of artefacts in a given environment, but they typically only include passive behaviour of the human;

3) unlike knowledge describing the pure physical behaviour of $P$ and $E$, knowledge related to the active behaviour of $U$ cannot straightforwardly be embedded in geometry;

4) knowledge that associates $P$ with different $U$ s and different $E$ s cannot be included;

5) simulations tend to be restricted to behaviour that is completely determined by one initial state. Consequentially, current simulation packages cannot cope with interventions that naturally occur in use processes.

Currently, our research focuses on the generation of kernel $U-P-E$ models to support modelling and forecasting use in conceptual design with a future system for generating resource-integrated models . The models are partly generated based on knowledge about use stored in ontologies, partly by the designer applying his or her own insights. For forecasting use processes, the knowledge in the models has to provide input for simulations. To overcome the problem that simulations cannot cope with multiple use processes, produced by a multitude of users, user behaviours, and environments, our idea is to apply multiple simulations based on scenarios, which can be associated with the $U-P-E$ system in order to pro- vide the starting conditions for simulations and forecasting. In the resource-integrated model, they are the carriers of the process knowledge that complements the physical behaviour processed in simulations and that usually cannot be captured in object-type models.

Instantiations of nuclei serve as building blocks in modelling the actors $U, P$ and $E$. The fact that nuclei can represent the physical characteristics of the actors in addition to their geometric and structural characteristics makes them attractive for modelling and simulation of use cases. Furthermore, it is also possible to consider them as function carriers, and to include them in a functional ontology. This ontology can incorporate scenarios consisting of situations known to occur in use processes.

In generating of models, we distinguish two cases:

a) conceptual design of a new product and

b) conceptual redesign of an existing product or of a product belonging to a known class of products.

In generating resource-integrated models, four activities are involved: modelling the user, modelling the product, modelling the environment, and modelling a scenario in which $U, P$ and $E$ appear as a system, involving situations that can be used as input for simulations.

To investigate the applicability of resource-integrated modelling in conceptual design, we have developed a nucleus-based model of an existing product, a pedal bin. The level of detailing of the object-type models of $U, P$ and $E$ corresponds to what we presumed to be appropriate in conceptual design. In our understanding it represents what the ontology can provide for us as a basis for a use-oriented redesign of an existing product - case (b). But it can also be

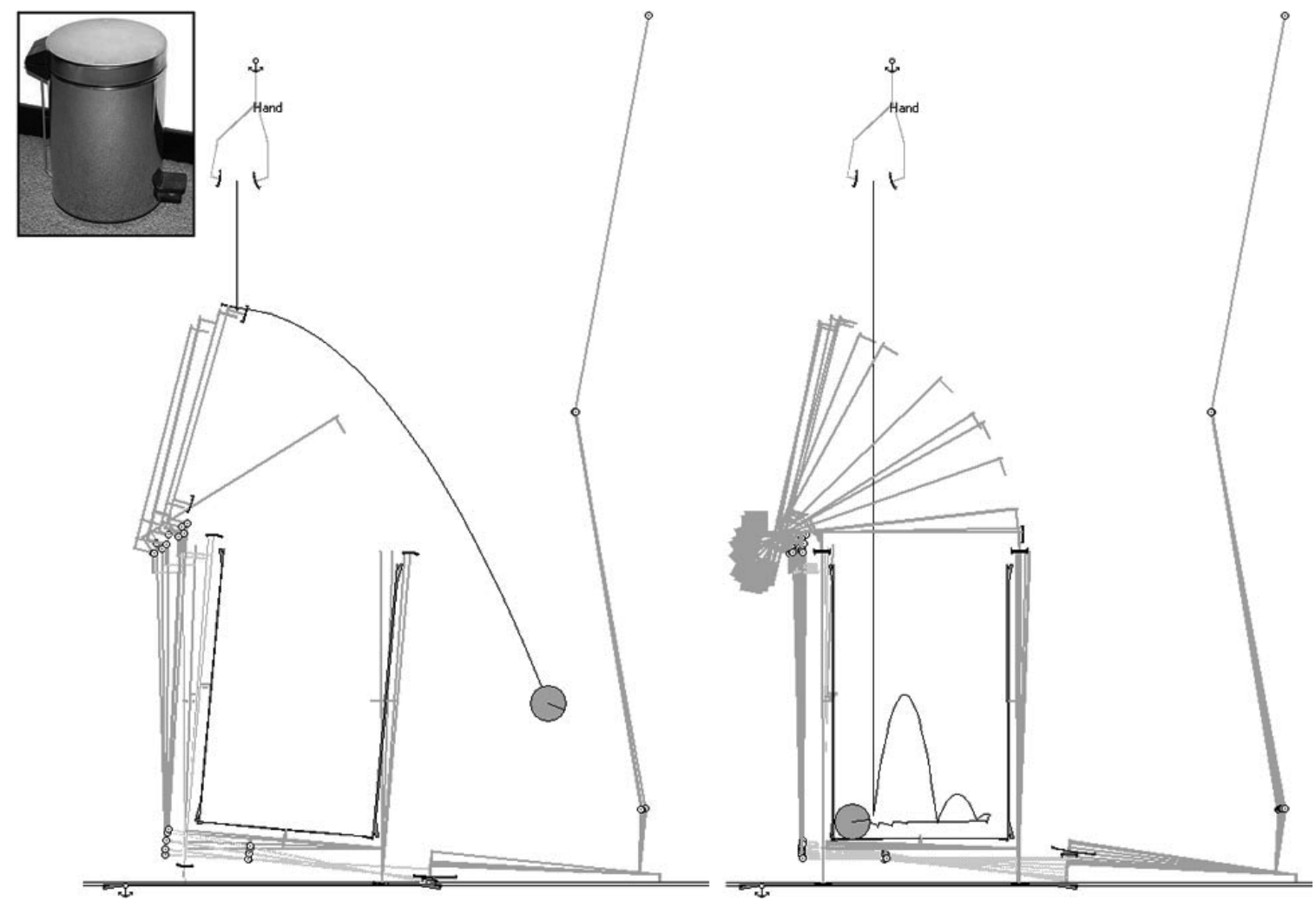

Fig. 2: (a) Conceptual model of a pedal-bin: simulation of a use scenario (left);

(b) Improved conceptual model of a pedal-bin: simulation of a use scenario (right) 
understood as an intermediate stage in the conceptual stage of a new product - case (a). We generated a qualitative description of a simple use scenario, disposal of a piece of garbage, which specifies the situations and the initial conditions for a simulation. The actual simulation was performed with MSC Working Model ${ }^{\circledR}$ 2D (WM2D). This package was also used to create most of the nucleus-based models of $U, P$ and $E$. Fig. 2a shows the initial resource-integrated conceptual model of the pedal bin in a simulation of its of usage. When the pedal is pressed, the bin starts to tumble and the lid tends to oscillate around its highest position. If the object is launched from the shown position, this behaviour of the bin prevents proper disposal. Fig. $2 b$ shows an improved conceptual design with a counterweight to ensure a more determined movement of the bin and the lid. The garbage now lands successfully inside the bin. This application case study demonstrates the method and issues of using nucleus-based modelling in conceptual design. The homogeneous representation of $U, P$ and $E$ enables us (1) to model the known use processes in the form of common scenarios and (2) to predict ad-hoc use processes based on simulations. Based on the results, an improved concept product can be realized on the level of detail that is typical for conceptual design. As a matter of course, we have to validate the findings for a wider range of products and use processes. In this respect it has to be made clear that the set-up of this tabletop research has apriori limitations in terms of an exhaustive validation of the hypothesis, for two reasons: (1) the generation or retrieval of known use processes was not based on a fully developed use ontology, nor on fully elaborated scenario-based modelling, and (2) the applied simulation technique, provisionally offered by the WM2D system, also posed limitations.

Despite these restrictions, the results revealed attractive prospects for the application of resource-integrated models to represent use-processes in conceptual design. Designers can anticipate the use process without consulting external knowledge sources about, and models of, users and environments, and without having to switch between object-type models and process-type models (including functional models). That is, processes involving simulation-based forecasting can be seamlessly included in the modelling environment, and even intervention-type interactions can be studied.

This development of a resource-integrated modelling environment has not yet reached the stage that its results can be applied or tested by designers in the form of computer support. However based on the currently available demonstrative examples, C-DET can give designers an impression of the future possibilities and can gather feedback about the actual need for the proposed form of design support. To go one step further, and provide designers insight into the potential of a full-featured system that combines the power of ontologies in combination with knowledge-intensive models, we will most probably require more advanced development of the two pillars of tool development that were discussed above. Progress is needed in particular in the following areas:

1) further development of the methodology and technology for capturing and processing knowledge related to the use of products,

2) further refinement of the fundamentals and methodology of modelling based on scenarios prescribing the use of products,
3) development of a dedicated simulation environment that can benefit from resource-integrated conceptual models.

\section{Extra functionality}

Once C-DET is put into use and starts to gain appreciation from design engineers, and once our research has resulted in some well-functioning fundaments, we can let C-DET grow in extent and in functionality. Then the following consideration will need to be taken into account. When a design engineer is working with C-DET, his work could be monitored by keeping track of the links he is using and by analysing the information he has inserted. Monitoring the design engineering work would give C-DET opportunities to support the designer by: Advising him where to store parts of his work; Offering him the tools he could use best at that time (give information about tools other users used in comparable cases); Advising him which aspect might need more attention (balanced comprehension). Therefore, we may have to consider monitoring the work of the designer. This has to be approached with care, since unwanted advice is annoying, and system users do not always want the computer to keep track of what they are doing.

The first form of design support we would like to incorporate in C-DET after it has survived its first stage as a knowledge portal and output-structuring tool, is helping the designer to come to a balanced comprehension of his design work. For this we need some tracking or monitoring tool to be able to follow the aspects the designer has given attention to. This part of design-support still needs a lot of research: How to monitor, how to evaluate, how to visualize the current status, etc. Furthermore, it might be valuable for designers if they are able to personalize their use of C-DET. This could mean for example that they are able to change certain ratings for their own specific use. This item needs a closer look to decide whether or not it is an option we want to deal with.

We could think of some future scenarios in which C-DET is very valuable for developing competitive products. Our first very immature ideas in this direction are about a C-DET-box (small suitcase) containing disk space, a Tablet PC, a scanner etc. The design engineer can plug in to the Internet from everywhere. Picture search, a shape search engine could be integrated and at in the very long term, some advanced input devices might be developed and incorporated. This section will be extended during the project.

\section{Conclusions and future results}

We have found criteria for selecting websites and tools based on their relevance and usability for industrial design engineering and thus for inclusion into C-DET. We aim to find aspect-specific criteria and design phase-specific criteria as well. The matrix we used as the entrance structure in the second prototype is preferred to the entrance structure based on design phases only, as used in the first prototype. The order of the aspects has to be adjusted in order to achieve a more logical array of aspects. We will also continue searching and evaluating other entrance structures, such as the structure based on the knowledge categorization of [9].

An introduction page in which the usage of the toolbox is explained may improve the ease of use. To keep C-DET up to 
date, developments of tools for design engineering will have be watched closely. One of these tools under development is discussed in this paper. A tool for forecasting the use process of a concept design offers added value to the designer that fits into C-DET very well. As we indicated, we can also include early prototypes of such tools in C-DET to give designers a preview of future developments and to provide the opportunity to give feedback to the researchers involved in new-tool development.

From our studies into the use of the two prototypes, we concluded that a phase-based structure alone does not facilitate optimal access to the tools and knowledge sources available within C-DET. Combined with the aspects in the matrix of the second prototype, most tasks for finding information, tools and knowledge sources were done within the set limit of ten minutes. However, to come to a balanced comprehension, an overview that the designer can apply to determine the imbalance in the completion of the task at hand, a more complete taxonomy of design aspects is needed. The aspect or combination of aspects that a designer is considering should be unambiguously designated in the entrance structure of C-DET, in order to be able to find the right tool for the right job. Therefore we will work on fine-tuning the list of design aspects or sub-aspects that we will use to make the contents of C-DET available.

\section{References}

[1] Vroom R. W. et al: Overview of the initiatives on the development of designer's toolkits. Procs. Design 2002. Zagreb: Faculty of Mechanical Engineering and Naval Architecture, 2002.

[2] Horváth I.: Conceptual Design: Inside and outside. Procs. EDIProD '2000. Zielona Góra: Technical University of Zielona Góra, 2000, p. 63-72.

[3] Roozenburg N. F. M., Eekels J.: Product design: fundamentals and methods. Chichester: Wiley, 1995.

[4] Vroom R.W.: Zicht op product- en procesontwikkeling. Doctoral dissertation, Delft: Delft University Press, 2001.

[5] Owen R., Horváth I.: Towards Product-related Knowledge Asset Warehousing in Enterprises. Procs. TMCE 2002, Wuhan: HUST Press, 2002, p.155-170.

[6] García J., Restrepo J.: Context modelling in a collaborative virtual reality application as support to the design process. Procs. Design 2002. Zagreb: Faculty of Mechanical Engineering and Naval Architecture, 2002.

[7] Christiaans H., Restrepo J.: Designer conditioning by early representations. Procs. SKCF '01. Sydney: 2001.

[8] Knijnenburg R., Braak G.: Intermediate Technical Report: Developing C-DET. Report IDE 350. Delft: Delft University of Technology, Industrial Design Engineering, 2003.
[9] Nijhuis K.: Het programma van eisen als instrument in de praktijk. Internal graduation report, Delft University of Technology, Industrial Design Engineering, 1996.

[10] Eder W. E.: EDC Engineering Design and Creativity. Procs. workshop EDC, November 1995, series WDK nr. 24. Zürich: Heurista. 1995.

[11] Bauer C., Scharl A.: "Quantitive evaluation of Web site content and structure." Internet Research: Electronic Networking Applications and Policy, Vol. 10, (2000), No. 1, http://www.emerald-library.com.

[12] Koekkoek M., Leving J.: Weblink library for conceptual design engineering. Report IDE 350. Delft: Delft University of Technology, Industrial Design Engineering, 2003.

13] Van der Vegte W.F., Horváth I.: Consideration and modeling of use processes in computer-aided conceptual design: a state of the art review. Transactions of the SDPS, Vol. 6, (2002), No. 2, p. 25-59.

[14] Kitamura Y., Mizoguchi R.: "Ontology-based description of functional design knowledge and its use in a functional way server." Expert Systems with Applications. Vol. 24, (2003), No. 2, p. 153-166.

[15] Horváth I., Van der Vegte W. F: Nucleus-based product conceptualization - part 1: Principles and formalization. To be published in Procs. ICED 2003, Stockholm, 2003.

[16] Van der Vegte W. F. et al: Ontology-based modeling of product functionality and use - part 2: considering use and unintended behavior. Procs. EDIProD ,02, Zielona Góra, Technical University of Zielona Góra, 2002.

[17] Van der Vegte, W. F., Horváth, I.: Nucleus-based product conceptualization - part 2: Application in Designing for Use. Proc. ICED 2003, Stockholm, 2003.

[18] Rivera T., Mitiguy T. (last revisers): Working Model User's Manual, MSC Software Corporation, San Mateo, 2001.

Dr. Regine W. Vroom

phone: +31 152781342

fax: +31 152781839

e-mail: r.w.vroom@io.tudelft.nl

Ernest J. J. van Breemen M. Sc. e-mail: e.j.j.vanbreemen@io.tudelft.nl

Wilhelm F. (Wilfred) van der Vegte MTD e-mail: w.f.vandervegte@io.tudelft.nl

Industrial Design Delft

Delft University of Technology

Landbergstraat 15

2265 CL Delft, The Netherlands 